Volume 9, No.5, September - October 2020

International Journal of Advanced Trends in Computer Science and Engineering

Available Online at http://www.warse.org/IJATCSE/static/pdf/file/ijatcse71952020.pdf

https://doi.org/10.30534/ijatcse/2020/71952020

\title{
A Property Oriented Pandemic Surviving Trading Model
}

\author{
Dr. D. A. Oyemade ${ }^{1}$,Dr. A. A. Ojugo ${ }^{2}$ \\ ${ }^{1}$ Department of Computer Science, Federal University of Petroleum Resources, Effurun. Delta State, Nigeria \\ oyemade.david@fupre.edu.ng \\ ${ }^{2}$ Department of Computer Science, Federal University of Petroleum Resources, Effurun. Delta State, Nigeria \\ ojugo.arnold@fupre.edu.ng
}

\begin{abstract}
The COVID-19 pandemic produced adverse effects on the global economy. The devastating impact of COVID-19 or coronavirus on the prices of commodities such as oil and gas as well as foreign exchange (Forex) trading systems and currency pairs across the nations, resulted in the collapse of businesses and shutdown of the economy. It was also accompanied with great loss of fund for investors in stock and Forex due to the consequent failure of some stock and forex software systems and strategies which were designed without envisaging the great volatility of market prices caused by the coronavirus pandemic. This study focuses on the examination and analysis of the performance of some Forex systems in the period of the pandemic. It identifies failed and successful systems during the period of the pandemic and proposes a pandemic surviving trading model to address the problem of the effect of unforeseen disaster such as the pandemic on trading systems. The systems examines the properties and data of failed and successful systems and integrates the best properties in the proposed model to provide a model which is likely to survive any possible future pandemic. The results show that systems developed with the property oriented pandemic surviving trading model produced a significant increase in profit during the pandemic compared to the existing system.
\end{abstract}

Key words: Property oriented system, automated software, COVID-19, coronavirus, trading model, Forex, global economy

\section{INTRODUCTION}

Coronavirus disease of 2019 (COVID-19) started like a spark of fire in December 2019 from the city of Wuhan China [1][2][9]. Within the space of about three month, it soon became a wild fire spreading into the whole globe with devastating effects on human health, human life, nations' economy, commodities prices, production levels, cities transportation, international flights, social life, students' academic life, religious life, sports, financial markets, agriculture, as well as automated forex software systems.Although the root cause of the pandemic was initially uncertain, its effects were real and negative. The debilitating impact of COVID-19 prompted the World Health Organization (WHO), national governments and the academic communities to swing into various actions for the purpose of limiting the spread of the pestilence and its associated mortalityrate [3-6][10-11][17]. For many Forex automated software systems, especially for Forex systems that were successful prior to the period of the pandemic, the adverse impact of COVID-19 manifested their decline of performance and profitability, due to abnormal price volatilities in the financial market caused by pandemic [12]. If left unattended to, such systems would definitely and woefully fail, producing loss of fund and invested capital rather than profits.

This study examines and analyses the software properties and data of some automated Forex software systems that failed in the heat of the pandemic and during its entireperiod. The main focus of the study is on user defined properties as distinct from platform-defined and conventional properties, built into the MetaTrader programmable platform commonly employed for the development of Forex expert advisors for automated trading. It proposes property oriented trading model integrated with an adaptive component for survival and profitability during the period of a pandemic or any other unforeseen economy-impacting occurrence.

The remaining part of this article is structuredusing the following arrangement: we first examine the background of study, then review the previous and related works. This is followed by the methodology. Sequel to this, we present the model application, the results and discussion, and finally, the conclusion and future works.

\section{BACKGROUND OF STUDY}

Unexpected occurrences, natural disasters and high impact news have been known to bear much influence on the prices of currency pairs in the Forex market. For example, on $15^{\text {th }}$ of March 2020, in the heat of the COVID-19 pandemic, the Great Britain Pounds Sterling and the Japanese Yen(GBP/JPY) currency pair descended to all time lowest price of 123.900 since 2012. This is a typical instance of the effect of natural disaster or inadvertent globally impacting event on currency pairs and forex trading. The amount of money lost by forex traders to this single debility of currency values could be very enormous. As a result of this influence of events and incidences, researchers and stakeholders in the Forex industry have classified the main approaches to Forex trading into two: fundamental analysis and technical 
analysis. In fundamental analysis, traders monitor important events that are capable of overturning the stability of Forex market. Such events are arranged chronologically on a time and event chart, known as Forex calendars, usually released and publishedby Forex brokers on daily, weekly and monthly basis. These events are usually classified into low impact, medium impact and high impact events and they are associated with predicted values. Trading algorithms are based on the time and predicted impact of these events on a particular currency pair. Apart from GBP/JPY, many other currency pairs exist. Few examples are CAD/JPY, $\mathrm{CAD} / \mathrm{CHF}$, USD/CNH and GBP/CAD. In these symbols, CAD stands for Canadian Dollar, USD stands for United States Dollars, CHF stands for Swiss Franc and CNH stands for Chinese Yuan. Forex trading typically involves the prediction of the values of these currency pairsfor the purpose of buying or selling one of the currency to make profit. In technical analysis, technical indicators are used for trading decisions. Some examples of these are Stochastic Oscillator, Alligator and Bands.Many of these technical indicators are programmable and are integrated into the MetaTrader programmable platform. Programmers and software developers also design and implement custom technical indicators for trading [21-22].

In automated software trading systems, expert advisors, otherwise known as Forex robots are often used and developed with MetaQuote programming language, which resemble $\mathrm{C}$ in structure and syntax. The MetaQuote language has numerous properties and functions and it is very versatile for the development and automation of Forex trading. Common intrinsic properties of the MetaQuote Languageinclude Ask, Bid, Take Profit and Stop Loss, Examples of functions in MetaQoute programming language are OrderSend, OrderClose and OrderSymbol.

Classical approaches to software design and development include object oriented, feature oriented and service oriented development [23-25]. The concept of semantic oriented modelling of data for enterprise applications has also been proposed [36]. Property-oriented and model-oriented abstraction have been suggested in software engineering practice without any specific model.This paper's contribution, amongst others, is the introduction of a property oriented model capable of solving the problem of volatilities of prices and uncertainty or decline of profit in Forex system during a pandemic period or similar periods of natural or unnatural disaster or awkward global events.

\section{RELATED WORKS}

Many research works in literature on the COVID-19 pandemic centred on ascertaining its root cause,characteristics, on limiting the spread, minimizing its associated mortality rate, analysing its global economic impacts and developing drugs and vaccines for COVID-19 [2-4][13-14][18]. Some studies considered its impact on transportation and the viability of controlling the outbreak by limiting contacts through isolation centres [7-8] and detection through the application of computer vision in radiology as well as cellular wireless network [15-16]. Design and development of medical, electronic and mechanical equipment for handling COVID-19 through manufacturing collaboration is another focus of some studies [19-20]. In [25], the composition of web services with feature-oriented design patterns was proposed. Other studies concentrated on the analysis of social media news and its effects using machine learning [33], measurement of elearning performance and satisfaction during COVID-19 period [34] and the performance assessment of some deep learning networks for the investigation of the spread and death caused by COVID-19 [35].

Different and diverse approaches have been employed for the forecasting of Forex time series data. These include statistical methods such as Autoregressive Moving Average (ARMA) and Auto Regressive Integrated Moving Average (ARIMA). Deep learning models such as Recurrent Neural Network (RNN), Multi-Layer Perceptron (MLP), STLM as well asreinforcement learning (RL) [26-29], a branch of machine learning, have also been used for Forex forecasting. Optimization techniques for Forex forecasting include Particle swarm optimization and extreme learning machine combined with jaya optimization [30][32]. Fuzzy set theories have also been applied in research efforts to finding solution to the task of forecasting Forex market prices [31].

Few studies such as [10] and [12] considered adverse of effects of COVID-19 on the financial markets. However, to our knowledge, no study has proposed a property oriented model to address the negative effect of the pandemic on financial market automated software systems. This is a major contribution of this paper. The introduction of a property oriented approach to solving the profitability problem of the financial market automated software system is another contribution of this study.

\section{METHODOLOGY}

The experiments in this study were carried out using MetaQuote programming language installed on MetaTrader platform. This was deployed on a Commercial Network Services, New York, virtual private server running on Windows 2003 (x86) Enterprise Edition R2, with 20 GB disk space and $640 \mathrm{Mb}$ RAM size, through paid subscription. The proposed model focuses on the software properties, otherwise known as attributes or data. The properties of a successful Forex system that was profitable prior to the period of coronavirus pandemic were studied. The decline in their performance and their failure due to the pandemic was acknowledged and noted at the outset of the pandemic. Using 
the proposed property-oriented pandemic surviving model, new properties and groups of properties that could tackle and handle the pandemic were designed, implemented and tested for the period of the pandemic. The focus here was userdefined functional forex exchange properties. The performance of this system was compared to the existing systems for the period of the pandemic.

\subsection{Property Oriented Pandemic Surviving Trading Model}

Figure 1 shows the conceptual diagram of the proposed property oriented pandemic surviving trading model. Five phases constitute the model. These are the confirmation, identification, integration, implementation and evaluation phases.

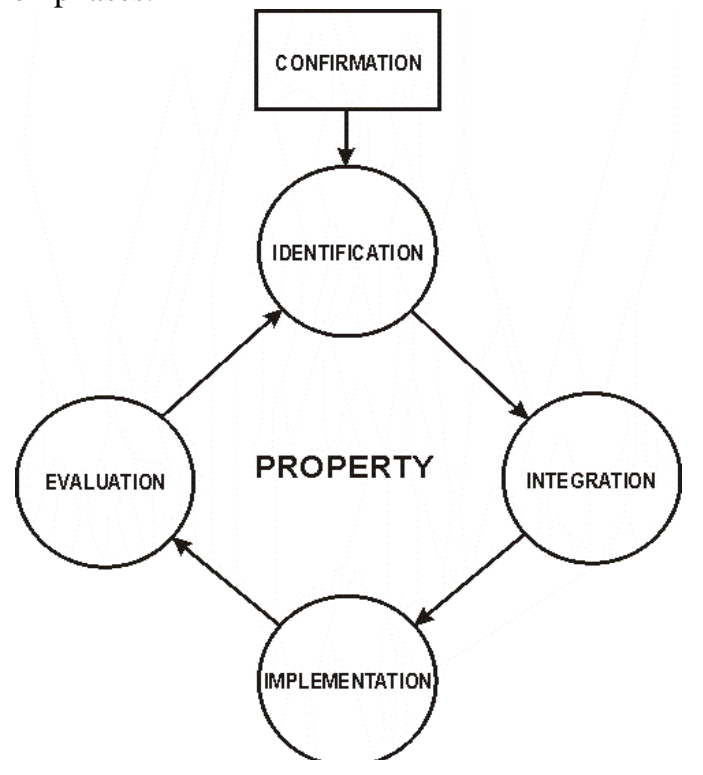

Figure 1: Conceptual Diagram of Property Oriented Pandemic Surviving Model

The application of the model begins from any point in time when a decline in the performance of a Forex system is discovered due to a pandemic or events that may have a long-term overwhelming effect on the market. Confirmation is the first phase of the model. The arrows signify the order.

\section{Confirmation}

Profitable Forex trading requires vigilance, considering the past historical adverse effects of events such as pandemic, natural disaster, change in national governments and high impact news on the Forex market prices. After such news or information concerning the events, any decline in the performance of operational Forex systems should be confirmed empirically. This confirmation is achieved by comparing the periodical profits of the Forex system at the pre-event period and the event kick-off period. The confirmation prompts the software architect, software developers and programmers to move to the next phase which is Identification.

\section{Identification}

In this phase, an examination of the various aspects of the software system is done, focusing on the identification of new user defined properties that are capable of solving the problem of decline in the system profit. This phase includes the development of appropriate algorithms or pseudo-code for the identified properties. Design of classes, methods, procedures or functions for the properties are done in this phase.

\section{Integration}

In the Integration phase, the new user-defined properties are incorporated into the software systems and combined with the intrinsic properties of the system. The algorithms and the pseudo-code are converted into MetaQuote language program codes. In this phase, the values of the essential properties are modified if necessary

\section{Implementation}

The implementation phase involves the deployment of the software system with the new properties on the MetaTrader platform for live trading, the connection of the system to the broker's server and, if necessary, to social trading websites. In this phase, multiple instances of the system can be deployed. In each instance, a limited number of the new properties are introduced to allow for the proper evaluation of the effect of each property. An instance of the implementation can also include changes in the values assigned to the user-defined properties to arrive at an optimal profit.

\section{Evaluation}

In the evaluation phase, a short assessment of the performance of the new system and its profitability is carried out. This can be done on daily, weekly or monthly basis. If the performance of the system is unsatisfactory, the cycle is repeated beginning with the identification of new properties.

\section{MODEL APPLICATION}

Figure2 shows the fairly consistent profit of a system for about six months prior to the commencement of the coronavirus pandemic.

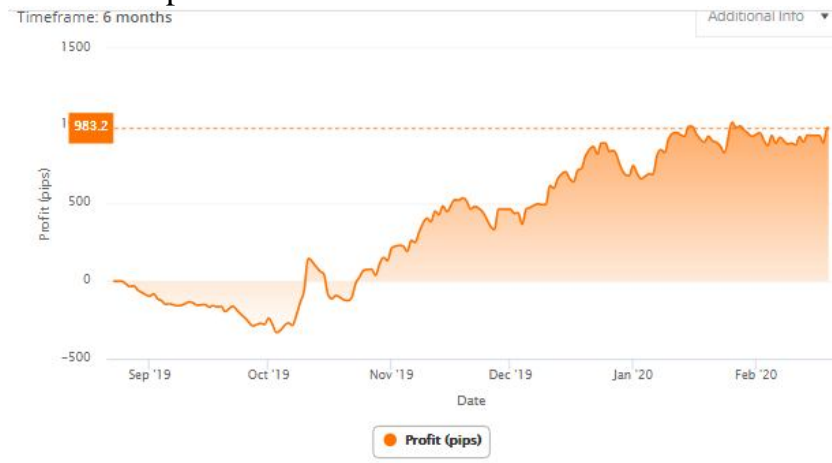

Figure 2:System Profit Chart for Pre-pandemic Period 
The signs of the adverse effects of COVID-19 pandemic on the system profit at the beginning of the epidemic is shown in Figure 3.The inconsistency and the declining profit occasioned by the pandemic as shown in Figure 3 is the confirmation. It signified that a more serious drop in profit would probably follow in the succeeding weeks and months if no action was taken to address issue.

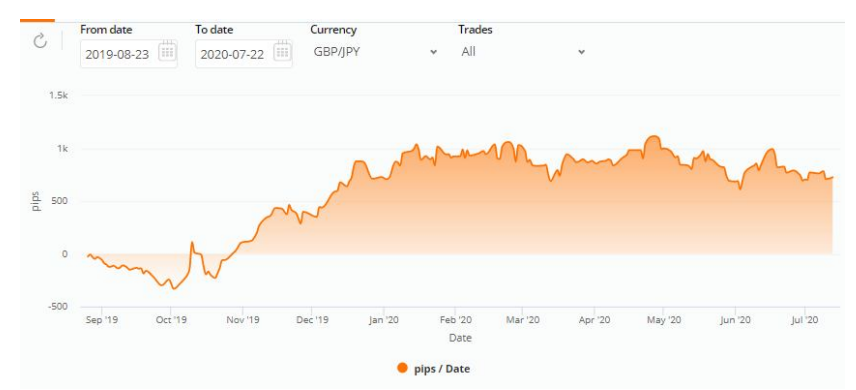

Figure 3: System Profit Chartat the Outset of the Pandemic

The properties identifiedfor possible profit consistency are: Adaptive Component, Sensitivity Component,Extra Modification, Adaptive System Activation Coefficient, Adaptive Trend Take Profit and Adaptive Swing Take Profit.

\section{Adaptive Component}

The adaptive component introduces a method of determining a period of volatility which is usually associated with high impact event or occurrence, capable of destabilizing the consistent performance of a Forex trading system.Selfupdating global variables are used to monitor M15 (15 minutes) support and resistance levels at three different times of the day: in the evening at the end of London trading session, at dawn and the current time. This difference in pips is converted to a coefficient by dividing it by 100 . A consistently high coefficient in the evening, at dawn and at current time implies volatility. A value greater than 1.1(110 pips) is considered to be high.Order placement is done using this coefficient. The pseudo-code for the adaptive component is shown below:

AdaptiveSystemActivationCoefficent=1.1; // normal. TwoTradesAdaptiveControlProfitAmount $=1$; /lensures a delay of 1 pip before placing a dissimilar order for a maximum order of two.

FromHourDawnM15Difference=6; // dawn starts from 6 GMT.

ToHourDawnM15Differnce=7; // dawn ends at 7 GMT.

FromHourPreviousNightM15Difference=18; // previous night starts from 18 GMT.

ToHourPreviousNightM15Difference=19; // previous night end at 19 GMT

CurrentM15MDifference $=$ M15MResistance Price M15SupportPrice; // capture this automatically.

IF Hour is between FromHourDawnM15Difference and ToHourDawnM15Differnce THEN
DawnM15MDifference $=$ M15MResistance Price M15SupportPrice; // capture this automatically.

IF Hour is between FromHourPreviousNightM15Difference and ToHourPreviousNightM15Difference THEN

PreviousNight $=$ M15MResistance Price - M15SupportPrice; // capture this automatically.

IF (DawnM15MDifference >=

AdaptiveSystemActivationCoefficentANDFromHourPrevious NightM15Difference >=

AdaptiveSystemActivationCoefficentANDCurrentM15MDiffe rence $>=$ AdaptiveSystemActivationCoefficentAND Basic

trading conditions are satisfied)

PlaceOrder;

\section{Sensitivity Component}

The sensitivity component places some constraints on the order placement by using the Ask and Bid prices for swing trading method to enable a quick placement of adaptive component orders. A region very close to the resistance level is defined by subtracting a very small fraction of the difference between the resistance and the support price for M15. Similarly, a region very close to the support level is defined by adding a very small fraction of the difference between the resistance and the resistance price for M15. The pseudo-code for the sensitivity component is shown below for a bearish order:

\section{IF ( (Ask<M15MResistance AND Ask>} M15MResistance*1/50) OR (Bid>M15MResistance*1/50

AND Bid<M15MResistance) AND Basic trading conditions are satisfied)

Place a bearish order

\section{Extra Modification}

The extra modification property is introduced for the purpose of increasing the probability of profitability whenever an active position has attained to a reasonable profit, since the sustenance of such a positive price cannot be guaranteed. Once a reasonable profit has been attained by an opened position but the market price is approaching a support or resistance level that may trigger a reversal, the priceof any possible pending order in the opposite direction is modified to a level very close to the support or resistance.This modification ensures that in case of price reversal, an opposite active order can be placed to trap the profit already attained. Below is the pseudo-code for extra modification for a bearish position:

IF ( profitsell >= ReasonableProfitAttained AND CurrentMarketPrice is close to SuportLevel) THEN Modify any bullish pending order 


\section{Adaptive System Activation Coefficient}

For the purpose of ascertaining the optimal value for the commencement of the adaptive component functionality, a value lower than 1.1 can beexperimented for the adaptive system activation coefficient. The value 0.95 was assigned to low adaptive system activation coefficient for distinction as shown below:

\section{LowAdaptiveSystemActivationCoefficent $=0.95$;}

\section{Adaptive Take Profit}

In Forex trading, a Take Profit value retrieves the profit once the market prices attains to that value. It is vital and relevant because a positive position can reverse to negative and eventually end up in a loss. Considering the risk and uncertainties that surrounds the Forex market especially in the period of price volatility, three special variables were introduced for the Take Profit property for the adaptive component and each of them is associated with the trend and swing market characteristics. Different values were set for low, medium and high take profits as shown below and used in three different systems.

$$
\begin{aligned}
& \text { LowAdaptiveTrendTakeProfit }=350 ; \\
& \text { LowAdaptiveSwingTakeProfit }=325 ; \\
& \text { MediumAdaptiveTrendTakeProfit }=450 ; \\
& \text { MediumLowAdaptiveSwingTakeProfit }=425 ; \\
& \text { HighAdaptiveTrendTakeProfit }=550 ;
\end{aligned}
$$$$
\text { HighLowAdaptiveSwingTakeProfit }=525 \text {; }
$$

The integration of all the identified properties into the MetaQuote programming language was carried out. The system was implemented on a MetaTrader platform for live trading during the period of the pandemic.The properties were distributed across five trading systems, including the system selected for the implementation of the proposed model. The systems code names are:Proposed Model System, Two in One, Low Trend, Fif Mode and Risk Rew.The distribution of the properties across the fivesystems is a means of measuring the strength of each property. Evaluation was carried by analysing the periodical profits of the systems used for the model implementation. Necessary properties values were adjusted within the first six weeks of the pandemic with feedback to the identification phase of the model. Table 1 shows the profit analysis of the five systemsfor the first six weeks of the pandemic. The profits shown in Table 1 are not taken as final but they are indicative of which of the systems are promising.
Table 1: Evaluation of Six Weeks Profit Analysis for Four Implemented Systems

\begin{tabular}{|l|c|}
\hline System Code Name & Profit \\
\hline Proposed Model System & 228 \\
\hline Two in One & 402 \\
\hline Low Treed & -87 \\
\hline Fif Mode & -129 \\
\hline Risk Rew & 368 \\
\hline
\end{tabular}

\section{RESULTS AND DISCUSSION}

Table 2 shows the properties selected for the proposed model. These includes: adaptive component, extra modification, adaptive system activation coefficient, low adaptive trend take profit and low adaptive swing take profit. None of these properties was used for the existing system. The experiments ran for four months in the heat of the pandemic period, from 15 th March to $22^{\text {nd }}$ July 2020. It can be deduced from Table 2 that while the existing model barely made a small profit of 36 pips for the period of the pandemic, the proposed property oriented model survived the pandemic with significantly high profit of 687 pips, a several multiple of the profit recorded by the existing model.

Table 2: Properties of the Proposed Model Compared to the Existing Model

\begin{tabular}{|l|c|c|}
\hline \multicolumn{1}{|c|}{ PROPERTIES } & \multicolumn{2}{c|}{ SYSTEMS } \\
\cline { 2 - 3 } & $\begin{array}{c}\text { Proposed } \\
\text { Model } \\
\text { System }\end{array}$ & $\begin{array}{c}\text { Existing } \\
\text { System }\end{array}$ \\
\hline Adaptive Component & $\checkmark$ & \\
\hline Extra M odification & $\checkmark$ & \\
\hline $\begin{array}{l}\text { AdaptiveSystemActivationCoef } \\
\text { ficient }\end{array}$ & $\checkmark$ & \\
\hline $\begin{array}{l}\text { Low Adaptive Trend Take } \\
\text { Profit }\end{array}$ & $\checkmark$ & \\
\hline $\begin{array}{l}\text { Low Adaptive Swing Take } \\
\text { Profit }\end{array}$ & $\checkmark$ & \\
\hline PANDEMIC PERIOD PROFIT & 687 & 36 \\
\hline
\end{tabular}


The system profit charts for the existing model and the proposed property oriented pandemic surviving trading model is shown in Figure 4.

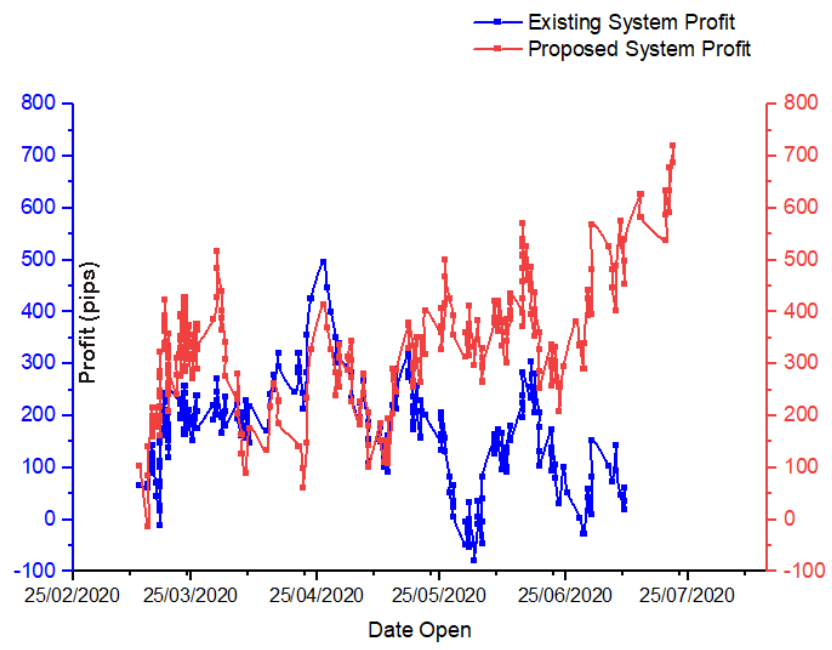

Figure 4: Profit Charts for the Existing System and the Proposed Property Oriented Pandemic Surviving Trading Model

The results of the proposed model, based on its performance, was matched with the results of other systems implemented with varied properties during the period of the pandemic for the purpose of assessing the strength of each properties. Table 3 shows the profits of four other systems and the featured properties of systems using a property matrix. Comparing Table 3 with Table 2, it can be seen that medium adaptive trend take profit properties and medium adaptive swing take profit properties are very profitable having been associated with Two in One and Risk Rew system that are also evidently promising. In addition, the sensitivity component property of Risk Rew system is profitable. Table 3 also shows that low adaptive trend take profit is undesirable having produced a loss at the end of the investigation period and being the only property that differentiates this system from the system of the proposed model. Low Trend system with high adaptive trend take profit and high adaptive swing take profit produced a huge loss. By implication, these two properties associated with this failed system are classified as adverse properties. Figure 5 illustrates the sharp difference in the performances of low trend system, the existing system and the proposed properties oriented pandemic surviving trading model's system.

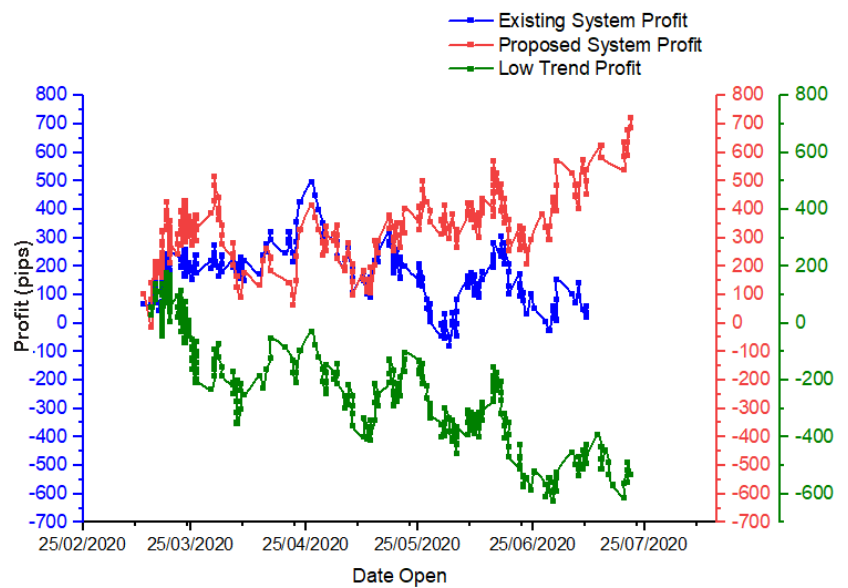

Figure 5: Comparative Charts for the Existing system, Proposed model and Low Trend system

Table 3Property Matrix for Property Oriented Pandemic Surviving Model

\begin{tabular}{|l|c|c|c|c|c|c|}
\hline \multicolumn{2}{|c|}{ PROPERTIES } & \multicolumn{5}{c|}{ SYSTEMS } \\
\hline & $\begin{array}{c}\text { Proposed } \\
\text { Model } \\
\end{array}$ & $\begin{array}{c}\text { Existing } \\
\text { System }\end{array}$ & $\begin{array}{c}\text { Two in } \\
\text { One }\end{array}$ & $\begin{array}{c}\text { Low } \\
\text { Trend }\end{array}$ & $\begin{array}{c}\text { Fif } \\
\text { Mode }\end{array}$ & $\begin{array}{c}\text { Risk } \\
\text { Rew }\end{array}$ \\
\hline Adaptive Component & $\checkmark$ & & $\checkmark$ & $\checkmark$ & $\checkmark$ & $\checkmark$ \\
\hline Sensitivity Component & & & & & & $\checkmark$ \\
\hline Extra M odification & $\checkmark$ & & & & $\checkmark$ & \\
\hline AdaptiveSystemActivationCoefficient & $\checkmark$ & & $\checkmark$ & $\checkmark$ & & $\checkmark$ \\
\hline Low Adaptive Trend Take Profit & $\checkmark$ & & & & $\checkmark$ & \\
\hline Low Adaptive Swing Take Profit & $\checkmark$ & & & & & \\
\hline Medium Adaptive Trend Take Profit & & & $\checkmark$ & & & $\checkmark$ \\
\hline Medium Adaptive Swing Take Profit & & & $\checkmark$ & & & $\checkmark$ \\
\hline High Adaptive Trend Take Profit & & & & $\checkmark$ & & \\
\hline High Adaptive Swing Take Profit & & & & $\checkmark$ & & \\
\hline LowAdaptiveSystemActivationCoefficient & & & & & $\checkmark$ & \\
\hline PANDEMIC PERIOD PROFITS & 687 & 36 & 605 & -464 & -73 & 506 \\
\hline
\end{tabular}




\section{CONCLUSION AND FUTURE WORK}

From the view point of property orientation, this work proposes a Forex trading model that can survive a highly volatile and inadvertent event such as the COVID-19 pandemic. It identifies user-defined properties required for a successful automated Forex trading software system, subjected to a pandemic, to include adaptive component, extra modification, adaptive system activation coefficient, low adaptive trend take profit and low adaptive swing take profit properties. This paper also highlights some undesirable system properties such as high adaptive swing and trend take profits. The results of live foreign exchange trading during four months of the pandemic period shows that the system developed with the proposed properties oriented pandemic surviving trading model produced a profit greater than a multiple of 19 compared to an existing system that was successful before the period of the pandemic but left to run without any modification or change. The proposed model shows the need for owners and developers of automated Forex trading software systems to be proactive at the outset of a pandemic or any global economy-impacting event that may result in abnormal volatility of the market prices. The proposed model is handy for the effective handling of such events.

Future works will focus on further research on profitable model properties and their effects on system profits, especially on the long-term effect of isolating a number of new user-defined properties during live Forex trading.

\section{REFERENCES}

1. Harapan Harapan andNaoya Itoh et al, "Coronavirus disease 2019 (COVID-19): A literature review", Journal of infection and public health, 13(5), pp.667-673, 2020

2. Heng Li and Shang-Ming Liu et al, "Coronavirus disease 2019 (COVID-19): current status and future perspectives", International journal of antimicrobial agents, 55(5), 105951, 2020

3. Muhammad Adnan Shereen andSuliman Khan et al, "COVID-19 infection: Origin, transmission, and characteristics of human coronaviruses", Journal of advanced research, 24, pp.91-98, 2020

4. John E. Ataguba, "COVID-19 Pandemic, a War to be Won: Understanding its Economic Implications for Africa", Applied health economics and health policy, 18(3), pp.325-328, 2020

5. Kabiru Saidu Musa and Rabiu Maijama'a et al, "COVID-19 Pandemic, Oil Price Slump and Food Crisis Nexus: An Application of ARDL Approach", Open Access Library Journal, 7, pp.119, 2020

6. Pedro Baqui and Ioana Bica et al, "Ethnic and regional variations in hospital mortality from COVID-19 in Brazil: a cross-sectional observational study" The Lancet. Global health, 8(8), pp.e1018-e1026, 2020

7. Joel Hellewell and Sam Abbott et al,"Feasibility of controlling COVID-19 outbreaks by isolation of cases and contacts", The Lancet. Global health, 8(4), pp.e488-e496, 2020

8. Emmanuel Mogaji "Impact of COVID-19 on transportation in Lagos, Nigeria. Transportation Research Interdisciplinary Perspectives", 6, 100154, 2020

9. Mingzuan Xie, M. and Qiong Chen, "Insight into 2019 novel coronavirus - An updated interim review and lessons from SARS-CoV and MERSCoV", International journal of infectious diseases : IJID : official publication of the International Society for Infectious Diseases, 94, pp.119-124, 2020

10. Peterson Ozili and Thankom Arun, "Spillover of COVID-19: Impact on the Global Economy", University Library of Munich, Germany, MPRA Paper 99317, 2020

11. S. Kaushik and S. Kaushik et al, "The Indian perspective of COVID-19 outbreak", Virusdisease, 31(2), pp.1-8, 2020

12. M. K. Mishra,The World after COVID-19 and its impact on Global Economy, ZBW - Leibniz Information Centre for Economics, Kiel, Hamburg, 2020, available at https://www.econstor.eu/bitstream/10419/215931/1/ MKM\%20PAPER\%20FOR\%20COVID.pdf

13. Xiaowei Li and Manman Geng et al, "Molecular immune pathogenesis and diagnosis of COVID19", Journal of pharmaceutical analysis, 10(2), pp.102-108, 2020

14. Giovanni Volpicelli and Luna Gargani,"Sonographic signs and patterns of COVID-19 pneumonia", Ultrasound $\quad J$ 12, 22, 2020

15. R. Punia and L. Kumar et al, "Computer Vision and Radiology for COVID-19 Detection", International Conference for Emerging Technology (INCET), Belgaum, India, pp.1-5, 2020

16. A. R. Alsaeedy and E. K. P. Chong, "Detecting Regions At Risk for Spreading COVID-19 Using Existing Cellular Wireless Network Functionalities", in IEEE Open Journal of Engineering in Medicine and Biology, vol. 1, pp.187-189, 2020

17. Catrin Sohrabi and Zaid Alsafi et al, "World Health Organization declares global emergency: A review of the 2019 novel coronavirus (COVID19)" International journal of surgery (London, England), 76, pp.71-76, 2020 
18. Mark Eisenstadt and ManoharanNiaz Ramachandran et al, "COVID-19 Antibody Test/Vaccination Certification: There's an App for That", IEEE Open Journal of Engineering in Medicine and Biology, vol. 1, pp.148-155, 2020

19. Abas Johar Jinia and Noora Sunbul et al, "Review of Sterilization Techniques for Medical and Personal Protective Equipment Contaminated With SARS-CoV-2", IEEE Access, vol. 8, pp.111347-111354, 2020

20. Weiming. Shen, Chen Yang and Liang Gao,"Address business crisis caused by COVID19 with collaborative intelligent manufacturing technologies", IET Collaborative Intelligent Manufacturing, vol. 2, no. 2, PP.96-99, 6, 2020

21. Farias Nazário and Rodolfo Toríbio et al, "A literature review of technical analysis on stock markets", The Quarterly Review of Economics and Finance, Elsevier, vol. 66(C), pages pp.115-126, 2017

22. Yoke Leng Yong, David C.L. Ngo and Yunli Lee, "Technical Indicators for Forex Forecasting: A Preliminary Study", Advances in Swarm and Computational Intelligence, 6th International Conference, ICSI 2015 held in conjunction with the Second BRICS Congress, CCI 2015, Beijing, China, Proceedings, Part III, Springerpp.25-28, 2015

23. Marvin Ferber, Thomas Rauber and Sascha Hunold,Combining Object-Oriented Design and SOA with Remote Objects over Web Services, 2010Eighth IEEE European Conference on Web Services, IEEE, pp.93-90

24. Diana Allam, Hervé Grall and Jean-Claude Royer,From Object-Oriented Programming to Service-Oriented Computing: How to Improve Interoperability by Preserving Subtyping, WEBIST 2013 - 9th International Conference on Web Information Systems and Technologies, Aachen, Germany, PP.169-173, hal-00800153

25. Vishnuvardhan Mannava and $\mathrm{T}$ Rames, "Composite Design Pattern For FeatureOriented Service Injection And Composition Of Web Services For Distributed Computing Systems With Service Oriented Architecture", International Journal of Web \& Semantic Technology (IJWesT) Vol.3, No.3, pp.73-84, 2012

26. Emam Ahmed,Optimal artificial neural network topology for foreign exchange forecasting, ACMSE 46: Proceedings of the 46th Annual Southeast Regional Conference on XXMarch 2008, pp. 63-68,

27. João Carapuço, Rui Neves and Nuno Horta, "Reinforcement learning applied to Forex trading", Applied Soft Computing Journal 73, Elsevier RV, pp.783-794, 2018
28. MehreenRehman, Gul Muhammad Khan and S. A. Mahmud, Foreign Currency Exchange Rates Prediction using CGP and Recurrent Neural Network, 2014 International Conference on Future Information Engineering, ScienceDirect, IERI Procedia 10, pp. $239-244$

29. Lina Ni andYujie Li et al, Forecasting of Forex Time Series Data Based on Deep Learning, International Conference on Identification, Information and Knowledge in the Internet of Things, ScienceDircct, Procedia Computer Science 147 (2019) pp.647-652, 2019

30. Smruti Rekha Das, Debahuti Mishra and Minakhi Rout,"A hybridized ELM-Jaya forecasting model for currency exchange prediction", Journal of King Saud University - Computer and Information Sciences Volume 32, Issue 3, PP.345-366, 2020

31. Ludmila Dymova, Pave Sevastjanov and Krzysziof Kaczmarek,"A Forex trading expert system based on a new approach to the rule-base evidential reasoning", Expert Systems With Applications 51,pp.1-13, 2016

32. Georgios Sermpinis, Konstantinos Theofilatos et al, "Forecasting foreign exchange rates with adaptive neural networks using radial-basis functions and Particle Swarm Optimization", European Journal of Operational Research, Elsevier, vol. 225(3), pp.528-540, 2013

33. K. Dheeraj, "Analysing COVID-19 News Impact on Social Media Aggregation", International Journal of Advanced Trends in Computer Science and Engineering, Vol. 9, No. 3, May - June 2020, pp. $2542-2549$

34. DienaDwidienawatiand Sri BramatoroAbdinagoro et al, "Forced Shifting to e-Learning during the COVID-19 Outbreak: Information Quality, System Quality, Service Quality, and Goal Orientation influence to e-Learning Satisfaction and Perceived Performance", International Journal of Advanced Trends in Computer Science and Engineering, Vol. 9, No. 2, March - April 2020, pp. $1518-1525$

35. Shafaf Ibrahim and Saadi Ahmad Kamaruddin, et al, "Performance Evaluation of Multi-Layer Perceptron (MLP) and Radial Basis Function (RBF): COVID-19 Spread and Death Contributing Factors", International Journal of Advanced Trends in Computer Science and Engineering, Vol. 9, No. 1.4 2020, pp. 625 - 631

36. Khadija Alaouiand Mohamed Bahaj, "Semantic Oriented Data Modeling for Enterprise Application Engineering Using Semantic Web Languages", International Journal of Advanced Trends in Computer Science and Engineering, Vol. 9, No. 3, May - June 2020, pp. 3229 - 3236 